\title{
Molecular Characterization of Carbapenem-resistant, Colistin-resistant Klebsiella pneumoniae Isolates from a Tertiary Hospital in Jeonbuk, Korea
}

\author{
Tae Hee Lee ${ }^{1,2+}$, Minhyeon Cho ${ }^{1+}$, Jaehyeon Lee ${ }^{3,5}$, Joo-Hee Hwang ${ }^{4,5}$, Chang-Seop Lee ${ }^{4,5 *}$, Kyung Min Chung ${ }^{1,2,5 *}$ \\ ${ }^{1}$ Department of Microbiology and Immunology, Jeonbuk National University Medical School, Jeonju, Jeonbuk 54896, \\ Republic of Korea \\ ${ }^{2}$ Institute for Medical Science, Jeonbuk National University Medical School, Jeonju, Jeonbuk 54896, Republic of Korea \\ ${ }^{3}$ Department of Laboratory Medicine, Jeonbuk National University Medical School, Jeonju, Jeonbuk 54896, Republic of Korea \\ ${ }^{4}$ Department of Internal Medicine, Jeonbuk National University Medical School, Jeonju, Jeonbuk 54896, Republic of Korea \\ ${ }^{5}$ Research Institute of Clinical Medicine of Jeonbuk National University-Biomedical Research Institute of Jeonbuk National \\ University Hospital, Jeonju, Jeonbuk 54907, Republic of Korea \\ tThese authors equally contributed to this work.
}

\author{
Corresponding \\ Chang-Seop Lee, MD, $\mathrm{PhD}$ \\ Department of Internal Medicine, \\ Jeonbuk National University Medical \\ School, Jeonju, Jeonbuk 54896, \\ Republic of Korea \\ Phone : +82-63-250-2391 \\ Fax : +82-63-250-1660 \\ E-mail : lcsmd@jbnu.ac.kr \\ Kyung Min Chung, $\mathrm{PhD}$ \\ Department of Microbiology and \\ Immunology, Jeonbuk National \\ University Medical School, Jeonju, \\ Jeonbuk 54896, Republic of Korea \\ Phone : +82-63-270-3068 \\ Fax : +82-63-270-3066 \\ E-mail : kmin@jbnu.ac.kr \\ Received : July 29, 2021 \\ Revised : August 12, 2021 \\ Accepted : August 14, 2021
}

No potential conflict of interest relevant to this article was reported.

Copyright (C) 2021 Journal of Bacteriology and Virology

(C) This is an Open Access article distributed under the terms of the Creative Commons Attribution Non-Commercial License

(http://creativecommons.org/

license/by-nc/3.0/).
Klebsiella pneumoniae resistant to both carbapenem and colistin has become a major clinical challenge. Although such resistant isolates are rare, they have been sporadically reported from many areas of the world. To investigate the prevalence and resistant mechanisms to carbapenem-resistant, colistin-resistant $K$. pneumoniae among Korean patients, we selected these resistant $K$. pneumoniae from clinical isolates collected over a period of five years at a tertiary hospital in Jeonbuk, Korea. The minimum inhibitory concentration of a variety of antibiotics against the resistant isolates was determined by the macrodilution method or the E-test. PCR analysis was used to determine sequence types (STs) and identify the genes involved in resistance to carbapenem and colistin. In 338 K. pneumoniae clinical isolates, two exhibited both carbapenem and colistin resistance. The ST of these belonged to ST11 and ST258, the most prevalent STs with K. pneumoniae carbapenemases, but the isolates did not carry any prevalent carbapenem-hydrolyzing $\beta$-lactamase genes. The carbapenem resistance was mediated by loss of porins OmpK35 and/or OmpK36 associated with DHA-1 AmpC $\beta$-lactamase. The colistin resistance was caused by amino acid changes in PmrB, PmrC, PmrE, PmrK, and MgrB, including novel amino acid changes in PmrE and PmrK. We first reported novel carbapenem-resistant and colistin-resistant $K$. pneumoniae ST11 and ST258 with mutations within ompK35, ompK36, $m g r B$, and $p m r$ genes in Korea. The results suggest that noncarbapenemase-producing carbapenem-resistant, colistin-resistant $K$. pneumoniae might be prevalent in Korea, and that better measures are necessary to control the spread of the resistant pathogen.

Key Words: K. pneumoniae, Carbapenem, Colistin, Porin, pmr genes

\section{INTRODUCTION}

Klebsiella pneumoniae, a gram-negative bacterium, is an important human opportunistic pathogen that can cause serious hospital- and community-acquired diseases, including septicemia, pneumonia, and urinary tract infections (1). The emergence and dissemination of multidrug-resistant (MDR) and extremely drug resistant (XDR) 
K. pneumoniae are of great health concern in many countries (1). In particular, carbapenem-resistant $K$. pneumoniae (CRKP) was recognized as a serious threat worldwide because the isolates often were co-resistant to most antibiotics, such as penicillins, cephalosporins, fluoroquinolones, and aminoglycosides (2). The most prevalent mechanism of resistance to carbapenems has been associated with production of carbapenem-hydrolyzing $\beta$-lactamases, including $K$. pneumoniae carbapenemases (KPCs) (Ambler class A), metallo- $\beta$-lactamases (MBLs) (Ambler class B), and OXA-48-like $\beta$-lactamases (Ambler class D) $(2,3)$. Carbapenem resistance has been conferred primarily by carbapenemase production mediated by KPCs (4). Worldwide, the predominant KPC-producing K. pneumoniae isolates are of sequence types (ST) 11 and 258 ; ST11 prevails mainly in East Asia, especially in China, and ST258 is most common in North America, Latin America, and several Europe countries $(3,5)$. In addition, some studies described how non-carbapenemase-producing CRKP (nCP-CRKP) can occur through impaired permeability via loss of outer membrane porins, OmpK35 and OmpK36, linked with AmpC-type $\beta$-lactamases $(6,7)$.

Infection with CRKP has limited therapeutic options, but one of the available antimicrobials is colistin, a last resort antibiotic that acts by disrupting the anionic lipopolysaccharide (LPS) component of the gram-negative outer membrane. The most common mechanism for colistin resistance in $K$. pneumoniae is mediated by modification of the LPS through cationic substitution (8). The LPS modification depends on a large panel of regulatory genes and operons $(8,9)$. Regulatory genes include those encoding proteins involved in the PmrA/PmrB system, the PhoP/PhoQ system, and a negative regulator (MgrB) of the PhoP/PhoQ system; the pmrC gene, pmrE gene, and pmrHFIJKLM operon are associated with LPS modification. Recently, plasmid-mediated colistin-resistant mcrgenes were identified in Escherichia coli and K. pneumoniae (8). More worryingly, colistin-resistant KPC-producing K. pneumoniae has been reported sporadically from Korea, Greece, North America, and other European countries in the past few years $(8,10)$. Therefore, the emergence and spread of colistin resistance in CRKP have increased the importance of long-term surveillance and an approach to gain insight into the exact mechanisms responsible for the resistance.

Although a number of studies has reported the prevalence and mechanism of $K$. pneumoniae resistance to carbapenem or colistin, $K$. pneumoniae that is resistant to both antibiotics has not been well demonstrated despite the increase in colistin-resistant CRKP. In this study, we screened carbapenem-resistant, colistin-resistant $K$. pneumoniae in clinical isolates collected from a Korean tertiary hospital to investigate the prevalence and mechanisms of carbapenem-resistant, colistin-resistant $K$. pneumoniae in Korea. Two isolates with both carbapenem and colistin resistance were identified and analyzed for STs and the genes associated with resistance to these antibiotics. Although the isolates did not carry any prevalent carbapenem-hydrolyzing $\beta$-lactamase genes, such as KPCs and OXA-48-like $\beta$-lactamases, we identified mutations within the ompK35, ompK36, $m g r B$, and $p m r$ genes that have not been observed in carbapenem-resistant, colistin-resistant K. pneumoniae ST11 and ST258 in Korea.

\section{MATERIALS AND METHODS}

\section{Bacterial isolates and antimicrobial susceptibility}

From 2008 to 2010 and from 2015 to 2018, 338 clinical isolates of K. pneumoniae were obtained from a tertiary hospital in Jeonbuk, Korea (Jeonbuk National University Hospital Culture Collection for Pathogens). The primary identification and antimicrobial susceptibility test were performed with a VITEK-2 system (bioMérieux, Marcy l'Etoile, France). The minimum inhibitory concentration (MIC) of antibiotics was determined by the macrodilution method using 2 ml of cation-adjusted Muller-Hinton broth in a standard glass test tube according to Clinical and Laboratory Standards Institute guidelines (8, 11). The MIC also was measured using the E-test carried out on a Muller-Hinton agar plate according to the manufacturer's instructions (bioMérieux, Marcy l'Etoile, France; Liofilchem Inc., MA, USA). As a control, K. pneumoniae ATCC 13883 purchased from the American Type Culture Collection (ATCC, VA, USA) was used. 


\section{Multilocus sequence typing (MLST)}

MLST of $K$. pneumoniae isolates was performed with seven housekeeping genes ( $g a p A$, infB, $m d h$, pgi, phoE, rpoB, and ton $B$ ), according to a previously described protocol (12). Allele sequences and ST were assigned according to Institute Pasteur's MLST scheme for K. pneumoniae.

\section{Analysis of antimicrobial resistance-related genes by polymerase chain reaction (PCR) amplification and sequencing}

Whole-bacterial DNA was prepared from bacterial cells lysed by heating at $95^{\circ} \mathrm{C}$ for $10 \mathrm{~min}$. The colistin resistanceassociated genes ( $p m r A, p m r B, p m r C, p m r D, p m r E, p m r k, p h o P, p h o Q$, and $m g r B$ ) were amplified using specific primers (Table 1) (13-15). PCR amplification detection for plasmid-mediated $m c r$ genes was performed using previously described primers $(16,17)$. The presence of extended-spectrum $\beta$-lactamase and carbapenem-hydrolyzing $\beta$-lactamase genes, such as bla $a_{C \mathrm{TX}-\mathrm{M}}, b / a_{\mathrm{SHV}}, b / a_{\mathrm{TEM}}, b / a_{\mathrm{KPCS}}$, and bla $a_{\mathrm{OXA}-48}$, was screened as previously described (18-20). PCR analysis for the ompK35 and ompK36 genes, K. pneumoniae porin genes associated with carbapenem resistance, was carried using the following primers: ompK35 forward, 5'- GCCGCCCGCACTCCACGCTG-3'; ompK35 reverse, 5'-CAGAAAGTTATGGAGCCACG-3'; ompK36 forward, 5'-GTATCATATTCTTGTTGGATTA-3'; and ompK36 reverse, 5'-GAAAGTCATATCCATTAATCG-3'. PCR analysis was used with previously described primers to identify the genes encoding AmpC-type $\beta$-lactamases from the following six families (21): MOX (MOX-1, MOX-2, CMY-1, and CMY-8 to CMY-11), CIT (LAT-1 to LAT-4, CMY-2 to CMY-7, and BIL-1), DHA (DHA-1 and DHA-2), ACC, EBC (MIR-1T and ACT-1), and FOX (FOX-1 to FOX-5b). The amplified PCR products were confirmed by agarose gel electrophoresis, purified from the agarose gel, and sequenced. The nucleotide and deduced protein sequences were analyzed at the National Center for Biotechnology Information website (www.ncbi.nlm.nih.gov) using the Basic Local Alignment Search Tool (BLAST) program.

Table 1. Primers for $P C R$ amplification of the $p m r, m g r B$, $p h o P$, and $p h o Q$ genes

\begin{tabular}{|c|c|c|c|}
\hline Gene & Primer direction & Primer sequence & Reference \\
\hline \multirow{2}{*}{ pmrA } & Forward & CATTTCCGCGCACTGTCTGC & \multirow{2}{*}{14} \\
\hline & Reverse & CAGCTTTCAGTTGCAAACAG & \\
\hline \multirow{2}{*}{$p m r B$} & Forward & ACCTACGCGAAAAGATTGGC & \multirow{2}{*}{14} \\
\hline & Reverse & GATGAGGATAGCGCCCATGC & \\
\hline \multirow{2}{*}{$\begin{array}{l}\text { pmrc } \\
(e p t A)\end{array}$} & Forward & ATGTCGTTATTGACTCTGCGTC & \multirow{2}{*}{ this study } \\
\hline & Reverse & tcaaccagcctctctacatgg & \\
\hline \multirow{2}{*}{$p m r D$} & Forward & TAGATGCTTCATGACGCTCTCTC & \multirow{2}{*}{12} \\
\hline & Reverse & gtgttatttgccggcgtttgtc & \\
\hline \multirow{2}{*}{ pmrE } & Forward & ATGAAAATTACTATTTCCGGTAC & \multirow{2}{*}{ this study } \\
\hline & Reverse & ctaatcattaccaaacagatcg & \\
\hline \multirow{2}{*}{ pmrk } & Forward & cctcaggcaaagtggttc & \multirow{2}{*}{ this study } \\
\hline & Reverse & gccgtccgcgacgggacg & \\
\hline \multirow{2}{*}{$m g r B$} & Forward & CAGCCAGCGATGCCAGATTT & \multirow{2}{*}{13} \\
\hline & Reverse & CCTGGCGTGATTTTGACACGA & \\
\hline \multirow{2}{*}{ phop } & Forward & GAGCTTCAGACTACTATCGA & \multirow{2}{*}{14} \\
\hline & Reverse & GGGAAGATATGCCGCAACAG & \\
\hline \multirow{2}{*}{ phoQ } & Forward & ATACCCACAGGACGTCATCA & \multirow{2}{*}{14} \\
\hline & Reverse & CAGGTGTCTGACAGGGATTA & \\
\hline
\end{tabular}




\section{RESULTS AND DISCUSSION}

\section{Isolation of non-carbapenemase-producing carbapenem-resistant $K$. pneumoniae (nCP-CRKP) with colistin resistance}

CRKP has been frequently identified as multidrug-resistant (MDR), including the potential development of resistance to colistin, a last-line antibiotic, and is associated with high rates of morbidity and mortality worldwide (22). To investigate the prevalence and the detailed mechanisms of MDR K. pneumoniae with carbapenem and colitis resistance, we assessed carbapenem and colistin resistance from the $K$. pneumoniae isolates collected by a Korean tertiary hospital, Jeonbuk National University Hospital Culture Collection for Pathogens. Out of 338 K. pneumoniae isolates, two (0.6\%) were identified as both carbapenem and colistin resistance. Interestingly, a high rate of colistin resistance in CRKP was observed as previously reported $(23,24)$. Two $(22 \%)$ of nine CRKP possessed colistin resistance (Table 2 ); the colistin MIC was $\geq 16 \mu$ $\mathrm{g} / \mathrm{ml}$ using the broth culture macrodilution method, but control K. pneumoniae ATCC13883 was not resistant to colistin. The two colistin-resistant isolates, LTH36 and LTH51, were resistant to all tested $\beta$-lactam antibiotics, including carbapenems (ertapenem, imipenem, and meropenem) and broad spectrum $\beta$-lactams (ceftazidime, cefotaxime, ciprofloxacin, and cefpirome) (Table 2). Intriguingly, the MIC ( $\geq 32 \mu \mathrm{g} / \mathrm{ml}$ ) for imipenem was higher in the two isolates than those of most CRKP within our health system (imipenem MIC range, 1 to $4 \mu \mathrm{g} / \mathrm{ml}$ ) (25). In addition, the isolates were resistant to aztreonam and aminoglycoside antibiotics (amikacin and gentamicin) (Table 2). However, one isolate, LTH51, remained susceptible only to tigecycline, as previously reported in other colistin-resistant $K$. pneumoniae, although the other isolate, LTH36, was not resistant to tigecycline and trimethoprim/sulfamethoxazole (Table 2) $(22,26)$.

Since the isolates exhibited resistant phenotypes to a variety of $\beta$-lactam antibiotics, including carbapenems, we investigated the presence of extend-spectrum- $\beta$-lactamase (ESBL) and carbapenem-hydrolyzing $\beta$-lactamase genes using PCR analysis and nucleotide sequencing. For ESBL genes, bla blasHV-31 and b/aTEM-116 genes, and the LTH51 isolate revealed the presence of the blacT-M-3, blasHV-11, and blatEM-1 genes. For detection of genes associated with carbapenem resistance, we evaluated the most prevalent carbapenem-hydrolyzing $\beta$

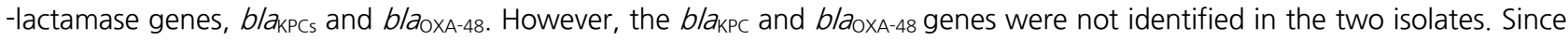
carbapenem resistance can occur with various types of carbapenemases, we screened for other carbapenem- hydrolyzing $\beta$ -lactamase genes with previously described primers to amplify the following genes (18): blaoxA-48-like including OXA-199 and

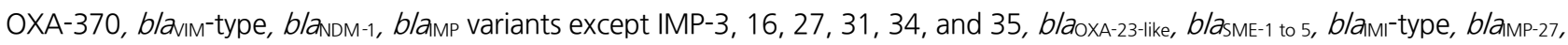

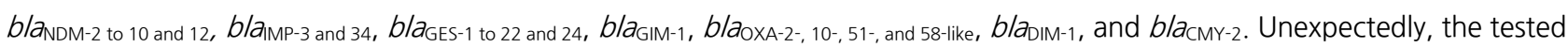
genes involved in carbapenem resistance were not detected in the two K. pneumoniae isolates, LTH36 and LTH51.

To investigate diversity, distribution, and relationship of our b/a $\mathrm{KPC}^{-}$and blaOXA-48-negative CRKP isolates with global isolates, we performed MLST. Interestingly, the STs of our colistin-resistant CRKP isolates LTH36 and LTH51 were ST11 and ST258,

Table 2. Antimicrobial susceptibility profiles of carbapenem-resistant, colistin-resistant K. pneumoniae isolates

\begin{tabular}{|c|c|c|c|c|c|c|c|c|c|c|c|c|c|}
\hline \multirow{2}{*}{ Isolate } & \multicolumn{13}{|c|}{$\mathrm{MIC}(\mu \mathrm{g} / \mathrm{ml})$} \\
\hline & ETPa & $\mathrm{IPM}^{\mathrm{b}}$ & MEM $^{a}$ & $\mathrm{CST}^{\mathrm{b}}$ & $\mathrm{AMK}^{\mathrm{a}}$ & GEN $^{b}$ & $\mathrm{TGC}^{\mathrm{b}}$ & $\mathrm{AZT}^{\mathrm{b}}$ & $C A Z^{b}$ & $C T X^{b}$ & CIPa & $\mathrm{CPR}^{\mathrm{a}}$ & STX ${ }^{a}$ \\
\hline ATCC13883 & $<0.25$ & 1 & $<0.25$ & 0.5 & 1 & $<0.25$ & 0.5 & $<0.25$ & 0.25 & $<0.25$ & $<0.25$ & $<0.25$ & $<0.25$ \\
\hline LTH36 & $\geq 32$ & $\geq 32$ & 8 & 16 & $\geq 256$ & $\geq 256$ & 1 & 128 & $\geq 256$ & $\geq 256$ & $\geq 32$ & 8 & 0.25 \\
\hline LTH51 & $\geq 32$ & $\geq 32$ & 24 & 32 & $\geq 256$ & $\geq 256$ & 1 & $\geq 256$ & $\geq 256$ & $\geq 256$ & $\geq 32$ & $\geq 256$ & $\geq 32$ \\
\hline
\end{tabular}

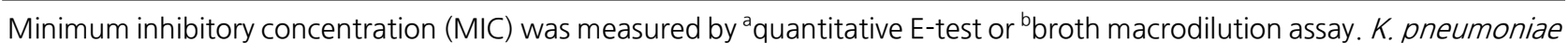
ATCC 13883 was used as a control strain. Antibiotic resistance was considered as an MIC of $>2 \mu \mathrm{g} / \mathrm{ml}$. ETP, ertapenem; IPM, imipenem; MEM, meropenem; CST, colistin; AMK, amikacin; GEN, gentamicin; TGC, tigecycline; AZT, aztreonam; CAZ, ceftazidime; CTX, cefotaxime; CIP, ciprofloxacin; CPR, cefpirome; STX, trimethoprim/sulfamethoxazole. 
respectively, which are the most global STs of $\operatorname{KPC}$-producing $\operatorname{CRKP}(3,5,27)$. Although the CRKP isolates with ST11 and ST258 were previously isolated in Korea, they carried KPC and showed a different susceptibility to colistin: the ST11 exhibited a colistin-resistant phenotype, but the ST258 did not $(10,28,29)$. Taken together, we first isolated $K$. pneumoniae ST11 and ST258 in Korea possessing both carbapenem and colistin resistance despite the absence of previously known carbapenem-hydrolyzing $\beta$-lactamase genes.

\section{Characterization of the carbapenem resistance in the nCP-CRKP isolates with colistin-resistance}

Because our two isolates were resistant to carbapenems without producing the most prevalent carbapenemases, we investigated an alternative mechanism for carbapenem resistance, which could result from mutation(s) in outer membrane major porins, OmpK35 and OmpK36, in association with AmpC-type $\beta$-lactamases. The deficiency of porin as a selective barrier would result in carbapenem resistance through reducing the permeability of porin; in addition, AmpC $\beta$-lactamases without carbapenemase activity could be involved in trapping carbapenems, which prevent the antibiotics from accessing their targets (6).

To test this possibility, we analyzed the sequences of porin genes, ompK35 and $0 m p K 36$, and the presence of bla $a_{A m p c}$ genes from six families: ACC, CIT, DHA, EBC, FOX, and MOX. In the sequence analysis of ompK35 and ompK36 compared with those of a control K. pneumoniae ATCC13883 (ompK35 and ompk36, GenBank accession no. CP040993.1), our LTH36 and LTH51 isolates revealed the lack of one or both of the two major porins. The LTH36 isolate revealed 1-bp deletion at position 54 and 1-bp insertion at position 419 of the ompk35 and ompk36 genes, respectively, which created amino acid changes and premature terminations after Gly-18 and Ser-139 in OmpK35 and OpmK36, respectively. In the LTH51 isolate, ompk36 sequencing reveled an ochre nonsense mutation after the start codon that could lead to the loss of OpmK36 porin, although there was wild type of the ompK35 gene. Furthermore, PCR and sequencing analysis of genes encoding $A m p C \beta$-lactamases showed that all of the $\mathrm{nCP}$-CRKP isolates with colistin resistance harbored the DHA-1 ampC $\beta$ -lactamase gene. These results suggest that the carbapenem resistance of the nCP-CRKP isolates LTH36 and LTH51 could be associated with loss of the major porins OmpK35 and/or OpmK36 and involved in DHA-1 AmpC $\beta$-lactamase production.

\section{Characterization of the colistin resistance in the nCP-CRKP isolates with colistin resistance}

To investigate the molecular mechanisms related to colistin resistance in the nCP-CRKP isolates with colistin resistance, we evaluated genetic mutations in the PmrA/PmrB and PhoP/PhoQ two-component regulatory systems, the PmrD connector of the two-component systems, the MgrB negative regulator of the PhoP/PhoQ, and LPS-modifying enzymes including PmrC, PmrE, and PmrK. Sequence analysis revealed amino acid variations in PmrB, PmrC, PmrE, PmrK, and MgrB compared with the colistin-susceptible $K$. pneumoniae reference strain, MGH78578 (Table 3). The same amino acid changes that were previously reported as contributing to colistin resistance were observed in PmrB (T246A and R256G) and PmrC $(\mathrm{C} 27 \mathrm{~F}, 1138 \mathrm{~V}$, and Q319R) from the two isolates $(8,30,31)$. Furthermore, we identified novel amino acid substitutions in PmrE (V17I, A33S, H68Q, T105A, A165E, D172N, A274G, N354D/A, E371D, and T373A) and PmrK (M114L, V117I, R372K, and G462R) from the LTH36 or LTH51 isolates, although more studies are required to prove a connection between the mutations and colistin resistance. Interestingly, although the two $K$. pneumoniae isolates belonged to different STs, there were the amino acid changes at the same position in PmrB, PmrC, PmrE, and PmrK: PmrB, T246A, and R256G; PmrC, C27F, I138V, and Q319R; PmrE, N354D/A and T373A; PmrK, M114L, V117I, and R372K. In the mgrB gene analysis of the two isolates, insertion sequences (ISs) were found in all isolates and caused insertional inactivation of the MgrB protein by missense and frameshift mutations, which resulted in amino acid residue and terminational changes after Leu-17 and Cys-39 of MgrB from the LTH36 and LTH51 isolates, respectively. However, PmrA, PmrD, PhoP, and PhoQ of the isolates were identical to the wild-type proteins of the reference strain K. pneumoniae MGH78578, and plasmid-mediated 
Table 3. Sequence types and genetic mutations in carbapenem-resistant, colistin-resistant $K$. pneumoniae isolates

\begin{tabular}{ccccccc}
\hline Isolate & ST & PmrB & PmrC & PmrE & PmrK & MgrB \\
\hline \multirow{2}{*}{ LTH36 } & \multirow{2}{*}{11} & T246A, & C27F, I138V, & V17I, A33S, H68Q, T105A, A165E, & M114L, V117I, & Frameshift (IS) \\
& & R256G & Q319R & D172N, A274G, N354D, E371D, T373A & R372K, G462R， & \\
LTH51 & \multirow{2}{*}{258} & T246A, & C27F, 1138V, & N354A, T373A & M114L, V117I, & Frameshift (IS) \\
& R256G & Q319R & R372K & C \\
\hline
\end{tabular}

The insertion sequences (ISs) were inserted after nucleotide position 51 and 117 of $m g r B$ (GenBank accession number KF852760.1) from the LTH36 and LTH51, respectively, and were identical to the followed regions: mgrB IS of LTH36, accession no. CP066855.1, region 2271513 to 2272285; mgrB IS of LTH51, accession no. MW650888.1, region 93977 to 94744. The reference GenBank accession number for $p m r A, p m r B, p m r C(e p t A), p m r D, p m r E, p m r k$, phoP, and phoQ gene is CP000647.1

colistin resistance genes, $m c r-1$ to $m c r-9$, were not detected in the isolates. These results suggest that the amino acid changes in PmrB, PmrC, PmrE, PmrK, and MgrB of the colistin-resistant CRKP isolates might be associated with colistin resistance.

In conclusion, we detected a high rate ( $22 \%, 2$ of 9$)$ of colistin resistance among CRKP although two $(0.6 \%)$ K. pneumoniae isolates with carbapenem and colistin resistance were found in 338 clinical isolates collected by a Korean tertiary hospital. The two isolates belonged to ST11 and ST258, the most prevalent STs of KPC-producing CRKP, but these carbapenem resistances were related to truncating mutations in the major porin ompK35 and/or ompK36 genes associated with DHA-1 AmpC $\beta$-lactamase. In colistin resistance, these isolates carried novel and previously reported mutations in the $p m r B, p m r C$, $p m r E$, pmrk, and $m g r B$ genes that could confer colistin resistance (Table 3). Taken together, we identified and characterized the detail molecular mechanisms of carbapenem and colistin resistance in K. pneumoniae ST11 and ST258 isolate that have not been reported in Korea. These results suggest that colistin-resistant nCP-CRKP with novel mutations emerged in Korea and could spread throughout the country. In addition, this study showed the importance of judicious use of antibiotics with proper susceptibility analysis as well as continuous surveillance and strict infection control measures on colistin-resistant CRKP to prevent the resistant bacteria from becoming prevalent in the hospital setting.

\section{ABBREVIATION}

KPC, K. pneumoniae carbapenemase; CRKP, carbapenem-resistant K. pneumoniae; nCP-CRKP, non-carbapenemase-producing carbapenem-resistant $K$. pneumoniae; MDR, multidrug-resistant; MIC, minimum inhibitory concentration; MLST, multilocus sequence typing; ST, sequence type

\section{ACKNOWLEDGEMENTS}

We thank Hyeon Ju Lee of Jeonbuk National University for her experimental assistance. The clinical isolates for this study were provided by the Jeonbuk National University Hospital as the branch of National Culture Collection for Pathogens (NCCP). This work was supported by National Research Foundation of Korea (NRF) grants (2019R1F1A1056056 and 2020M3A9G3080282) funded by the Korean government (MSIT) and the Fund of Biomedical Research Institute, Jeonbuk National University Hospital.

\section{CONFLICT OF INTEREST}

No potential conflict of interest relevant to this article was reported. 


\section{REFERENCES}

1) Navon-Venezia S, Kondratyeva K, Carattoli A. Klebsiella pneumoniae: a major worldwide source and shuttle for antibiotic resistance. FEMS Microbiol Rev 2017:41:252-75.

2) Lee $\mathrm{CR}$, Lee JH, Park KS, Kim YB, Jeong BC, Lee SH. Global dissemination of carbapenemase-producing Klebsiella pneumoniae: Epidemiology, genetic context, treatment options, and detection methods. Front Microbio/2016;7:895.

3) Pitout JD, Nordmann P, Poirel L. Carbapenemase-Producing Klebsiella pneumoniae, a key pathogen set for global nosocomial dominance. Antimicrob Agents Chemother 2015:59:5873-84.

4) Munoz-Price LS, Poirel L, Bonomo RA, Schwaber MJ, Daikos GL, Cormican M, et al. Clinical epidemiology of the global expansion of Klebsiella pneumoniae carbapenemases. Lancet Infect Dis 2013:13:785-96.

5) Qi Y, Wei Z, Ji S, Du X, Shen P, Yu Y. ST11, the dominant clone of KPC-producing Klebsiella pneumoniae in China. J Antimicrob Chemother 2011;66:307-12.

6) Hamzaoui Z, Ocampo-Sosa A, Fernandez Martinez M, Landolsi S, Ferjani S, Maamar E, et al. Role of association of OmpK35 and OmpK36 alteration and b/aEsBL and/or b/ampC genes in conferring carbapenem resistance among non-carbapenemase-producing Klebsiella pneumoniae. Int J Antimicrob Agents 2018;52:898-905.

7) Leavitt A, Chmelnitsky I, Colodner R, Ofek I, Carmeli Y, Navon-Venezia S. Ertapenem resistance among extendedspectrum-beta-lactamase-producing Klebsiella pneumoniae isolates. J Clin Microbio/2009;47:969-74.

8) Poirel L, Jayol A, Nordmann P. Polymyxins: Antibacterial activity, susceptibility testing, and resistance mechanisms encoded by plasmids or chromosomes. Clin Microbiol Rev 2017;30:557-96.

9) Groisman EA. The pleiotropic two-component regulatory system PhoP-PhoQ. J Bacterio/2001;183:1835-42.

10) Rhee JY, Park YK, Shin JY, Choi JY, Lee MY, Peck KR, et al. KPC-producing extreme drug-resistant Klebsiella pneumoniae isolate from a patient with diabetes mellitus and chronic renal failure on hemodialysis in South Korea. Antimicrob Agents Chemother 2010;54:2278-9.

11) Clinical and Laboratory Standards Institute (CLSI). Performance standards for antimicrobial susceptibility testing; 24th informational supplement. CLSI document M100-S24. Clinical and Laboratory Standards Institute, Wayne, PA, 2014.

12) Diancourt L, Passet V, Verhoef J, Grimont PA, Brisse S. Multilocus sequence typing of Klebsiella pneumoniae nosocomial isolates. J Clin Microbio/2005;43:4178-82.

13) Cheng HY, Chen YF, Peng HL. Molecular characterization of the PhoPQ-PmrD-PmrAB mediated pathway regulating polymyxin B resistance in Klebsiella pneumoniae CG43. J Biomed Sci2010;17:60.

14) Choi MJ, Kim S, Ko KS. Pathways Regulating the pbgPoperon and colistin resistance in Klebsiella pneumoniae strains. J Microbiol Biotechno/2016;26:1620-8.

15) Jayol A, Poirel L, Brink A, Villegas MV, Yilmaz M, Nordmann P. Resistance to colistin associated with a single amino acid change in protein PmrB among Klebsiella pneumoniae isolates of worldwide origin. Antimicrob Agents Chemother 2014;58:4762-6.

16) Kieffer N, Royer G, Decousser JW, Bourrel AS, Palmieri M, Ortiz De La Rosa JM, et al. mcr-9, an inducible gene encoding an acquired phosphoethanolamine transferase in Escherichia coli, and it's origin. Antimicrob Agents Chemother 2019;63:e00965-19.

17) Wang $X$, Wang $Y$, Zhou $Y$, Li J, Yin W, Wang S, et al. Emergence of a novel mobile colistin resistance gene, mcr-8, in NDM-producing Klebsiella pneumoniae. Emerg Microbes Infect 2018;7:122. 
18) Mlynarcik $P$, Roderova $M$, Kolar M. Primer evaluation for PCR and its application for detection of carbapenemases in Enterobacteriaceae. Jundishapur J Microbio/2016:9:e29314.

19) Schechner V, Straus-Robinson K, Schwartz D, Pfeffer I, Tarabeia J, Moskovich R, et al. Evaluation of PCR-based testing for surveillance of KPC-producing carbapenem-resistant members of the Enterobacteriaceae family. J Clin Microbiol 2009;47:3261-5.

20) Tofteland S, Haldorsen B, Dahl KH, Simonsen GS, Steinbakk M, Walsh TR, et al. Effects of phenotype and genotype on methods for detection of extended-spectrum-beta-lactamase-producing clinical isolates of Escherichia coli and Klebsiella pneumoniae in Norway. J Clin Microbio/2007:45:199-205.

21) Pérez-Pérez FJ, Hanson ND. Detection of plasmid-mediated AmpC beta-lactamase genes in clinical isolates by using multiplex PCR. J Clin Microbio/2002;40:2153-62.

22) Bogdanovich T, Adams-Haduch JM, Tian GB, Nguyen MH, Kwak EJ, Muto CA, et al. Colistin-resistant, Klebsiella pneumoniae carbapenemase (KPC)-producing Klebsiella pneumoniae belonging to the international epidemic clone ST258. Clin Infect Dis 2011:53:373-6.

23) Rojas LJ, Salim M, Cober E, Richter SS, Perez F, Salata RA, et al. Colistin resistance in carbapenem-resistant Klebsiella pneumoniae: Laboratory detection and impact on mortality. Clin Infect Dis 2017:64:711-8.

24) van Duin D, Doi Y. Outbreak of colistin-resistant, carbapenemase-producing Klebsiella pneumoniae: Are we at the end of the road? J Clin Microbio/2015:53:3116-7.

25) Marchaim D, Chopra T, Pogue JM, Perez F, Hujer AM, Rudin S, et al. Outbreak of colistin-resistant, carbapenemresistant Klebsiella pneumoniae in metropolitan Detroit, Michigan. Antimicrob Agents Chemother 2011;55:593-9.

26) Tóth A, Damjanova I, Puskás E, Jánvári L, Farkas M, Dobák A, et al. Emergence of a colistin-resistant KPC-2-producing Klebsiella pneumoniae ST258 clone in Hungary. Eur J Clin Microbiol Infect Dis 2010;29:765-9.

27) Ko KS. Antibiotic-resistant clones in Gram-negative pathogens: presence of global clones in Korea. J Microbiol 2019;57:195-202.

28) Livermore DM. Current epidemiology and growing resistance of gram-negative pathogens. Korean J Intern Med 2012:27:128-42.

29) Yoo JS, Kim HM, Yoo Jl, Yang JW, Kim HS, Chung GT, et al. Detection of clonal KPC-2-producing Klebsiella pneumoniae ST258 in Korea during nationwide surveillance in 2011. J Med Microbio/2013;62:1338-42.

30) Cheong HS, Kim SY, Seo J, Wi YM, Peck KR, Ko KS. Colistin resistance and extensive genetic variations in PmrAB and PhoPQ in Klebsiella Pneumoniae isolates from South Korea. Curr Microbio/2020;77:2307-11.

31) Jaidane N, Bonnin RA, Mansour W, Girlich D, Creton E, Cotellon G, et al. Genomic insights into colistin-resistant Klebsiella pneumoniae from a Tunisian Teaching Hospital. Antimicrob Agents Chemother 2018;62:e01601-17. 